\title{
Learning-Induced Plasticity Regulates Hippocampal Sharp Wave-Ripple Drive
}

\author{
Gabrielle Girardeau, Anne Cei, and Michaël Zugaro \\ Collège de France, Center for Interdisciplinary Research in Biology, and Centre National de la Recherche Scientifique, Unité Mixte de Recherche 7241, \\ F-75005 Paris, France
}

Hippocampal sharp wave-ripples (SPW-Rs) and associated place-cell reactivations are crucial for spatial memory consolidation during sleep and rest. However, it remains unclear how learning and consolidation requirements influence and regulate subsequent SPW-R activity. Indeed, SPW-R activity has been observed not only following complex behavioral tasks, but also after random foraging in familiar environments, despite markedly different learning requirements. Because transient increases in SPW-R rates have been reported following training on memory tasks, we hypothesized that SPW-R activity following learning (but not routine behavior) could involve specific regulatory processes related to ongoing consolidation. Interfering with ripples would then result in a dynamic compensatory response only when initial memory traces required consolidation. Here we trained rats on a spatial memory task, and showed that subsequent sleep periods where ripple activity was perturbed by timed electrical stimulation were indeed characterized by increased SPW-R occurrence rates compared with control sleep periods where stimulations were slightly delayed in time and did not interfere with ripples. Importantly, this did not occur following random foraging in a familiar environment. We next showed that this dynamic response was abolished following injection of an NMDA receptor blocker (MK-801) before, but not after training. Our results indicate that NMDA receptor-dependent processes occurring during learning, such as network "tagging" and plastic changes, regulate subsequent ripplemediated consolidation of spatial memory during sleep.

Key words: consolidation; hippocampus; learning; memory; regulation; ripples

\section{Introduction}

Sharp wave-ripple (SPW-R) complexes are a prominent endogenous hippocampal activity pattern arising during quiet behavioral states (Buzsáki et al., 1992; Ylinen et al., 1995; Csicsvari et al., 2000). Massive synchronized excitation from CA3 pyramidal cells discharges distributed subsets of CA1 pyramidal cells and interneurons, the combined activity of which results in highfrequency oscillations $(150 \sim 250 \mathrm{~Hz}$ ripples $)$ in the CA1 pyramidal layer (Ylinen et al., 1995; Memmesheimer, 2010; Schomburg et al., 2012). During SPW-Rs, hippocampal cell assemblies reinstate network activity patterns encoded during exploration. As a rat explores its environment, hippocampal pyramidal cells selectively discharge in specific spatial locations, coding for the position of the animal in space (O'Keefe and Dostrovsky, 1971; Wilson and McNaughton, 1993). Successive activation of these

Received Oct. 7, 2013; revised Feb. 25, 2014; accepted Feb. $27,2014$.

Author contributions: G.G. and M.Z. designed research; G.G. and A.C. performed research; G.G. and M.Z. analyzed data; G.G. and M.Z. wrote the paper.

This work was supported by the International Human Frontiers Science Program Organization (CDA0061/2007C), by European Project NeuroProbes (IST-027017), and by a fellowship from the Fondation pour la Recherche Médicale. We thank S.I. Wiener, L. Roux, E. Cerasti, S. Sara, K. Benchenane, S. Doutremer, M.-A. Thomas, Y. Dupraz, S. Rateau, A. Fruchart, P. Ruther, S. Kisban, and S. Herwik for advice and technical support.

The authors declare no competing financial interests.

Correspondence should be addressed to Michaël Zugaro, Collège de France, Center for Interdisciplinary Research in Biology (CIRB), 11, place Marcelin Berthelot, 75005 Paris, France. E-mail: michael.zugaro@college-defrance.fr. G. Girardeau's present address: New York University Medical Center, Neuroscience Institute, New York, NY 10016. DOI:10.1523/JNEUROSCI.4288-13.2014

Copyright $\odot 2014$ the authors $\quad 0270-6474 / 14 / 345176-08 \$ 15.00 / 0$ "place" cells generates neuronal sequences representing spatial trajectories (Skaggs and McNaughton, 1996; Dragoi and Buzsáki, 2006; Diba and Buzsáki, 2008). These sequences are later replayed at accelerated rates during SPW-Rs (Skaggs et al., 1996; Nádasdy et al., 1999; Lee and Wilson, 2002; Davidson et al., 2009). Because the ripple frequency is propitious for LTP induction (Buzsáki, 1984), this suggested an implication of SPW-Rs in memory (Buzsáki, 1989). Indeed, sleep and rest SPW-Rs following training are critical for spatial memory consolidation (Girardeau et al., 2009; Ego-Stengel and Wilson, 2010), possibly mediating intrahippocampal reinforcement and hippocampo-neocortical transfer of newly acquired memory traces for long-term stabilization (Marr, 1971; Buzsáki, 1989; Girardeau and Zugaro, 2011). Although SPW-Rs also occur during non-exploratory awake states, such as brief pauses during exploration, these SPW-Rs do not appear to be involved in memory consolidation, but in different, complementary functions, such as memory-guided decision making (Foster and Wilson, 2006; Diba and Buzsáki, 2007; Carr et al., 2011; Jadhav et al., 2012).

While there is now ample evidence that sleep and rest SPW-Rs are involved in spatial memory consolidation, how learning and consolidation requirements influence and regulate SPW-R dynamics remains unclear. Indeed, the hippocampal circuit consistently generates SPW-Rs during sleep and rest, independently of prior behavior, and SPW-Rs have been observed following both complex behavioral tasks and routine exploration of familiar environments, despite markedly different learning requirements. 
A

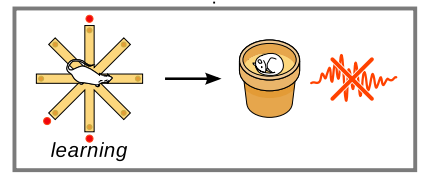

paired sessions

B
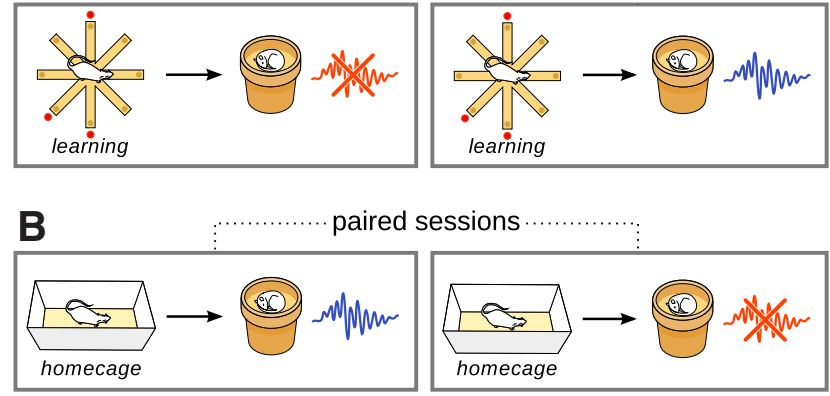

C
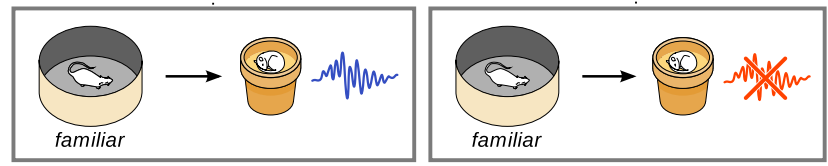

D

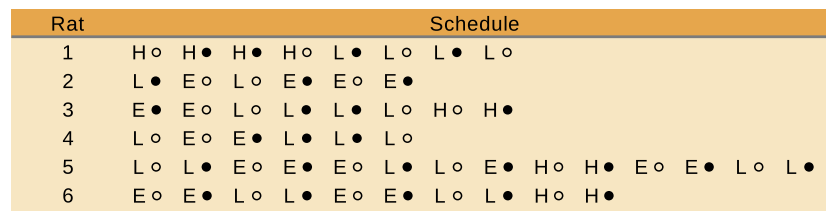

Figure 1. Experimental schedule. $\boldsymbol{A}-\boldsymbol{C}$, Behavioral conditions and stimulation protocols were alternated in a pseudorandom manner. For each behavioral condition ( $\boldsymbol{A}$, training on the maze; $\boldsymbol{B}$, home cage; $\boldsymbol{C}$, exploration of a familiar arena), stimulation protocols were paired for comparison (red crossed ripple icon, detection - stimulation; blue intact ripple icon, detection- delay-stimulation). $\boldsymbol{D}$, Experimental schedule for all rats. Because conditions and protocols were intermingled in the same rat, training on the maze was limited and discontinuous, preventing assessment of progressive changes in task performance. (H, home cage; $\mathrm{E}$, exploration of the familiar arena; L, learning the radial maze task; closed circle, immediate stimulation; open circle, delayed stimulation).

On the other hand, SPW-R occurrence rates increase following training on memory tasks (Eschenko et al., 2008; Ramadan et al., 2009). This raises the possibility that SPW-Rs may be regulated by different mechanisms in learning versus nonlearning conditions, i.e., when SPW-Rs underlie consolidation processes triggered by previous learning experience, versus when they constitute automatic, baseline activity patterns. We reasoned that interfering with ripples would then result in a dynamic response only in the former scenario, when SPW-Rs are necessary for memory consolidation and actively generated by a hippocampal network previously primed during behavior. We thus interfered with memory consolidation by selectively interrupting ripples (and putative associated replay) following training on a spatial reference memory task, and compared this to a control condition where rats foraged for food in a familiar environment. We then investigated the role of network plasticity processes induced during learning by pharmacological blockade of NMDA receptors during training.

\section{Materials and Methods}

Pretraining. Long-Evans male rats (250-300 g upon arrival) were maintained on food restriction to $85 \%$ of their normal weight. For the spatial memory task, rats were pretrained every weekday to find three food rewards at three fixed locations on an eight-arm radial maze, as previously described (Girardeau et al., 2009). Each session consisted of three trials on the maze, separated by 3 min rest periods when the rat was secluded in a flowerpot in the center of the maze. On each trial, the rat was removed from the maze as soon as it found the three rewarded arms, or after a maximum of 3 min of exploration. Visual cues suspended on the walls of the room served as spatial reference cues. In the exploratory locomotor task, rats were pretrained to forage for randomly scattered food in a circular arena ( $20 \mathrm{~min}$ per day for $3 \mathrm{~d}$ ). All experiments were conducted in accordance with institutional (Centre National de la Recherche Scientifique Comité Opérationnel pour l'Ethique dans les Sciences de la Vie) and international (National Institutes of Health guidelines) standards and national legal regulations (certificat no. 7186, Ministère de l'Agriculture et de la Pêche).

Surgery. The rats were bilaterally implanted either with two independently movable 9 -site or 16-site silicon probes or four independently movable single wires. Bipolar stimulation electrodes were implanted in the ventral hippocampal commissural pathway (mediolateral, $\pm 1.1 \mathrm{~mm}$; anteroposterior, $-1.3 \mathrm{~mm}$; dorsoventral, $-3.8 \mathrm{~mm}$ relative to bregma). During recovery from surgery $(\geq 3 \mathrm{~d})$, the rats received food and water $a d$ libitum. The recording electrodes were then progressively adjusted until they reached the CA1 pyramidal cell layer where ripples were recorded.

Training and MK-801 injections. Two days before testing on the radial maze, the spatial configuration of the baited arms was changed to require new learning. Recordings were performed during sleep and rest following either (1) three trials on the eight-arm radial maze, (2) locomotion in the familiar circular arena, or (3) rest in the home cage. To block NMDA receptors, the rats received intraperitoneal injections of MK-801 either 15 min before training on the maze (test) or immediately following training (control). Complementary control saline injections were performed in both conditions.

Recordings and stimulations. Brain signals were preamplified, amplified $1000 \times$ (L8, Neuralynx), acquired, and digitized using two synchronized Power1401 systems (Cambridge Electronic Design). As previously described (Girardeau et al., 2009), online ripple detection (threshold crossing on the bandpass-filtered signal) automatically triggered a singlepulse $(0.5 \mathrm{~ms})$ ventral hippocampal commissural stimulation that canceled further development of the ripple (detection rate, $>83 \pm 2 \%$; false detection rate, $<19 \pm 2 \%$ ). Stimulation sessions took place in the familiar flowerpot and lasted $\leq 1 \mathrm{~h}$. The stimulation voltage was adjusted for each animal to the minimum value necessary to interrupt the ripples $(5-30 \mathrm{~V})$. The stimulation was either triggered at the onset of the ripple (test) or after a random delay $(80-120 \mathrm{~ms}$, control), leaving the ripple intact. The number of stimulations was limited to five per second. All experiments took place during the light cycle. Recordings were visualized and processed using NeuroScope and NDManager (Hazan et al., 2006; http://neuroscope.sourceforge.net; http://ndmanager. sourceforge.net).

Data analyses. Sleep stages [slow-wave sleep/rapid eye movement (SWS/REM)] were determined by automatic $k$-means clustering of the theta/delta ratio extracted from the power spectrograms during the episodes where the animal was immobile. All periods when the rat was moving or in REM sleep were discarded. The remaining periods were concatenated to yield single blocks of sleep and rest, during which each ripple triggered an (immediate or delayed) stimulation. Sessions containing $<600$ s of cumulated sleep/rest were discarded from subsequent analyses. We then plotted the cumulative number of stimulations against time, and computed the best-fit slope (linear regression, $y=a x$ ) and slope significance $(t$ test for regression slopes; $p$ values are reported using a color scale on Figs. 3, 5, 6). For each behavioral condition (radial maze, foraging, homecage), we compared the slopes and amplitudes between the two stimulation protocols (detection-stimulation vs detection-delay-stimulation). Critically, by comparing all stimulation protocols and behavioral conditions in the same animals and on consecutive days (or with at most 3 intervening days: consecutive days, $n=43 ; 1 \mathrm{~d}, n=3 ; 2 \mathrm{~d}$, $n=2 ; 3 \mathrm{~d}, n=1$ ), the experiment was carefully designed to minimize spurious differences in ripple detection rates due to variations in electrode placement or inter-individual variability. Two additional analyses were performed by grouping data for each animal (see main text). One outlier was removed from the MK-801 analysis (residual larger than expected from $95 \%$ confidence intervals). 


\section{Results}

Interfering with spatial memory consolidation by timed ripple interruption triggers a dynamic upregulation of SPW-R activity

Rats were trained on a hippocampusdependent spatial memory task, in which they searched for food rewards at three fixed locations on an eight-arm radial maze (Girardeau et al., 2009; Ramadan et al., 2009). Using timed single-pulse stimulations of the ventral hippocampal commissure, ripples were selectively suppressed to interfere with memory consolidation during sleep and rest episodes following training sessions (Girardeau et al., 2009, Ego-Stengel and Wilson, 2010). To determine whether this interference procedure would affect SPW-R dynamics, we measured SPW-R occurrence rates during sleep periods where ripples were automatically interrupted (test), and compared this to a matched control protocol where SPW-Rtriggered stimulations were delayed in time, permitting ripples to occur normally (Figs. 1A, 2A, B).

Critically, in this and all subsequent experiments, test and control stimulations were performed in the same animals and in close temporal proximity (Fig. 1) to rule out spurious intraindividual and interindividual variability in ripple occurrence and detection rates. Similarly, to allow comparisons between different learning paradigms (see below), it was essential to alternate between different behavioral conditions in the same animal. While this design precluded meaningful assessment of behavioral performance, it leveraged our previous study already establishing that ripple disruption interferes with memory consolidation on the radial arm task (Girardeau et al., 2009) and was thus purposely optimized for the study of SPW-R physiology.

We counted the cumulative number of SPW-Rs as a function of sleep and rest time (Fig. 2C), and measured the SPW-R occurrence rate as the slope of the linear regression $(y=a x)$. Comparison of SPW-R occurrence rates between test and control sessions (Fig. 3) showed a significant homeostatic-like immediate increase during the sleep periods where ripple activity was perturbed (paired $t$ test, $p=0.0068, n=12$ session pairs on 6 rats, $\mathrm{df}=11, t=-3.32)$. This did not depend on the order in which the stimulation protocols were performed ( $t$ test, $p=0.0631$, $n 1=n 2=6$ sessions, $\mathrm{df}=10, t=2.09)$. Besides, the response had a fast time course, as indicated by the fact that cumulative ripple counts for immediate versus delayed stimulations already started to diverge from the beginning of the sleep sessions (paired $t$ tests comparing curve tangent slopes at 15 s intervals, $p<0.05$ ).

We performed two additional analyses on grouped data. We first averaged for each rat the ripple occurrence rates over repeated experiments ("mean rates" method), counting the two stimulation protocols separately. This yielded a single pair of values (immediate vs delayed stimulations) for each rat. We compared the two stimulation protocols using a paired $t$ test. Note that in this procedure, individual sessions from single rats can no longer be paired, which results in a more conservative test. In the second analysis (mean ratios method), we determined for each session pair the ratio between the ripple occurrence rates in the immediate versus delayed stimulation sessions, then computed the mean ratio for each rat. We tested whether this was equal to 1 (equal ripple rates, null hypothesis) using a $t$ test. Both analyses confirmed our results on ungrouped data (mean rates: paired $t$ test, $p=0.0130, n=6, \mathrm{df}=5, t=-3.77$; mean ratios: $t$ test, $p=$ $0.0129, n=6$, df $=5, t=3.78$ ).

To rule out the possibility that the observed SPW-R upregulation could result from uncontrolled plasticity-inducing effects of the stimulation, we measured the slopes of the elicited field postsynaptic potentials and compared them in the beginning and end of a stimulation session. Stimulation during SPW-Rs did not induce detectable changes in network responses (paired $t$ tests; slopes: $p=0.95, n=10, \mathrm{df}=9, t=-0.069$; Fig. $4 A$; amplitudes: $p=0.38, n=10, \mathrm{df}=9, t=0.930)$. Furthermore, stimulations during ripples did not elicit greater network excitation than outside ripples (paired $t$ tests; slopes: $p=0.16$, $n=10, \mathrm{df}=9, t=-1.531$; Fig. $4 B$; amplitudes: $p=0.12, n=10$, $\mathrm{df}=9, t=-1.719)$. Thus, interference with ripples following training dynamically increased ongoing SPW-R activity.

Interestingly, when the rats remained in their home cages and were not exposed to the radial maze on the recording day (Fig. $1 B$ ), this response was no longer observed (data not shown; paired $t$ test, $p=0.6552, n=5$ session pairs on 4 rats, $\mathrm{df}=4$, $t=-0.48$; mean rates: paired $t$ test, $p=0.4610, n=4$, $\mathrm{df}=3$, $t=-0.84$; mean ratios: $t$ test, $p=0.3561, n=4, \mathrm{df}=3, t=1.08$ ), suggesting a role for hippocampal activity elicited during maze exploration.

\section{Mere spatial exploration is not sufficient to trigger dynamic SPW-R upregulation}

We then tested whether the increased ripple drive following maze exploration was specifically related to learning. Because even repetitive, stereotyped activation of place cells during behavior can trigger sequence reactivations in overtrained animals not engaged in cognitive tasks (Skaggs and McNaughton, 1996; Lee and Wilson, 2002), we tested whether simple exploratory locomotor activity would be sufficient to induce dynamic changes in SPW-R incidence upon ripple perturbation. We trained the rats to forage for food in a familiar arena (Fig. $1 C$; see Fig. $5 A$ ) to elicit hippocampal theta oscillations and activate place cell sequences without exposing the animals to novelty or triggering learning. Surprisingly, interrupting ripples during subsequent sleep and 
A

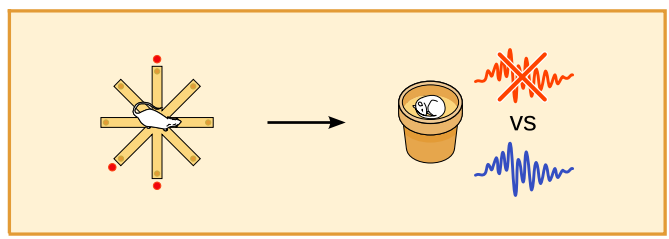

B

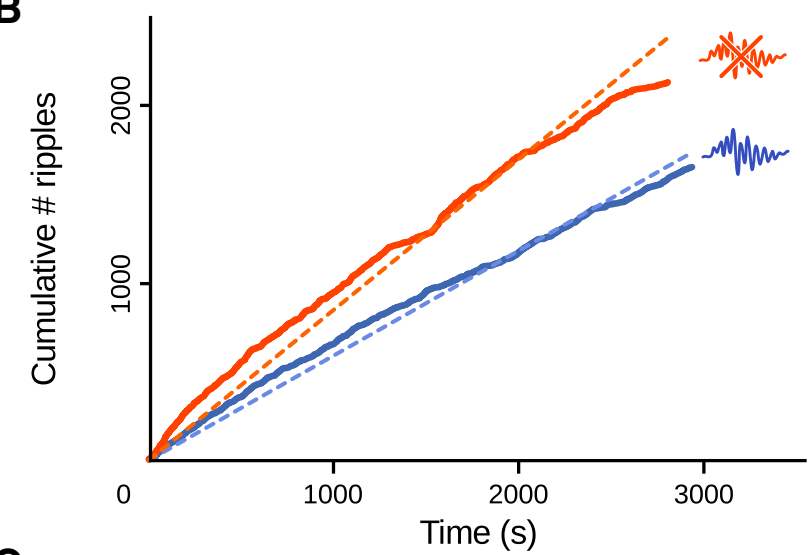

C

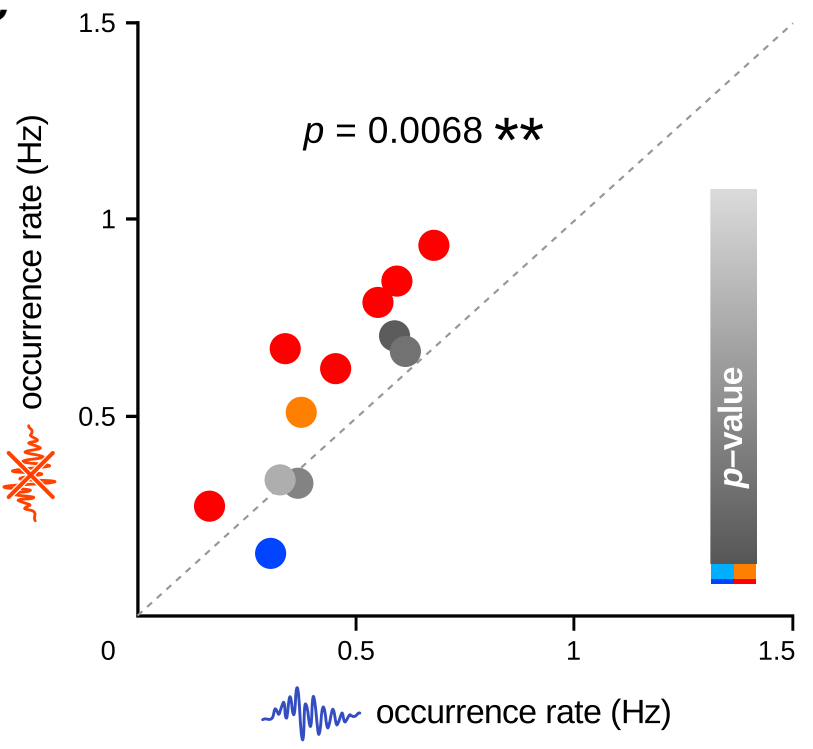

Figure 3. Interfering with memory consolidation by selective ripple suppression triggers dynamic increases in SPW-R incidence after learning. A, During sleep and rest following training on the radial maze task (baited arms, red spots), single-pulse stimulation of the ventral hippocampal commissure was triggered either immediately upon ripple detection, preventing further ripple development, or following a random delay $(80-120 \mathrm{~ms})$, leaving ripples intact (Fig. 2). $\boldsymbol{B}$, Example session pair. Ripples were counted as a function of time, during periods where they were either interrupted (orange curves) or left intact (blue curves). Ripple occurrence rates were computed as the slopes of the best-fit lines ( $y=a x$ dashed lines). C, SPW-R occurrence rates increased significantly in response to ripple suppression (paired $t$ test, $p=0.0068, n=12$ ). Each dot corresponds to one session pair. The color indicates the $p$ value for the comparison of the corresponding linear regression slopes, as illustrated in $\boldsymbol{B}$ ( $t$ tests: red and dark blue, $p<0.01$; orange and light blue, $p<0.05$; gray, $p>0.05$ ).

rest did not induce increased SPW-R occurrence rates (paired $t$ test, $p=0.5405, n=9$ session pairs on 4 rats, $\mathrm{df}=8, t=0.70$; Fig. $5 B, C$; mean rates: paired $t$ test, $p=0.6314, n=5$, df $=4$, $t=0.51$; mean ratios: $t$ test, $p=0.5974, n=5$, df $=4, t=-0.57)$. This rules out a substantial influence of mere recent spatial experience, and confirms that the increased SPW-R drive after training on the radial maze was specifically triggered by learning-
A

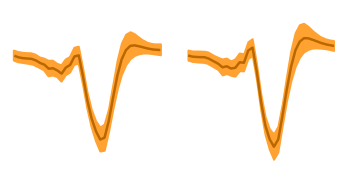

B
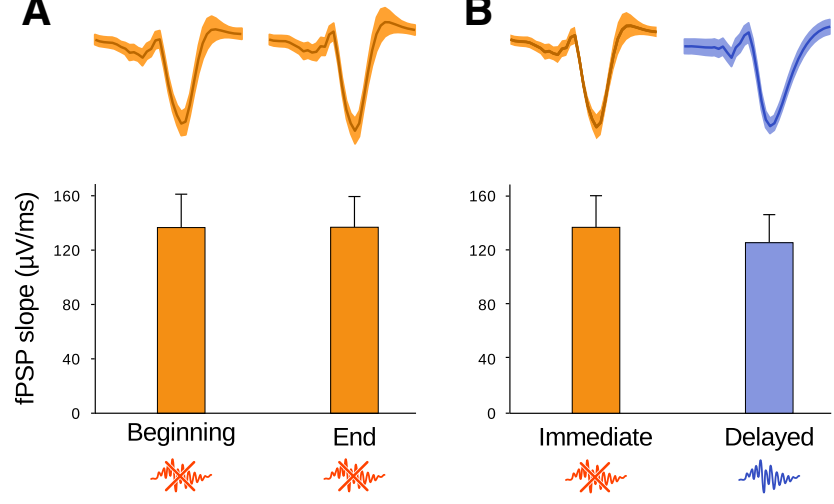

Figure 4. Stimulation during SPW-Rs does not induce detectable changes in network excitability or plasticity after learning. Slopes of field postsynaptic potentials (fPSPs) were measured during sleep and rest sessions following training on the radial maze. fPSP traces for one example session pair are shown in the top row (solid curve, mean; shaded area, SD). $A$, The fPSP slopes are not significantly different between the beginning and the end of the recording sessions (paired $t$ test, $p=0.95, t=-0.069, n=10, \mathrm{df}=9)$. $\boldsymbol{B}$, The fPSP slopes are not significantly different between immediate and delayed stimulation (paired $t$ test, $p=0.16, t=-1.531, n=10$, $\mathrm{df}=9$ )

related mechanisms elicited during the task, and preparing for subsequent consolidation.

Increased SPW-R drive is triggered by NMDA receptordependent mechanisms during learning, but not during sleep Candidate mechanisms for the postlearning increase in SPW-R drive include initial synaptic plasticity and network "tagging" during learning, which depend on the NMDA receptor and have been proposed to be crucial for setting the stage for memory consolidation (Frey and Morris, 1997; Lesburguères et al., 2011). To test this, we administered the noncompetitive NMDA receptor blocker MK- 801 to the rats before training $(0.05 \mathrm{mg} / \mathrm{kg}$, a dose known to impair learning but not consolidation; McLamb et al., 1990). Consistent with previous reports (McLamb et al., 1990), MK-801 injections did not alter locomotor activity (overall mean velocity: $6.9 \pm 0.6 \mathrm{~cm} / \mathrm{s}$ vs $6.4 \pm 0.4 \mathrm{~cm} / \mathrm{s}, t$ test, $p=0.55, n 1=24$ sessions, $n 2=41$ sessions, $\mathrm{df}=43, t=0.603$; mean velocity during movement: $19.7 \pm 0.6 \mathrm{~cm} / \mathrm{s}$ vs $18.1 \pm 0.6 \mathrm{~cm} / \mathrm{s}$, $t$ test, $p=0.08, n 1=24$ sessions, $n 2=41$ sessions, $\mathrm{df}=43$, $t=1.78$; immobility ratio: $0.57 \pm 0.02$ vs $0.59 \pm 0.02$, $t$ test, $p=0.63$, $n 1=24$ sessions, $n 2=41$ sessions, $\mathrm{df}=43, t=-0.48)$. Similarly, overall SPW-R characteristics were minimally affected by MK801: while amplitude increased by $4.7 \%$ ( $t$ test, $p=0.011)$, frequency and duration remained unchanged ( $t$ tests, $p=0.33$ and $p=0.51$ respectively). Systemic injections of MK- 801 abolished the dynamic response upon ripple perturbation during sleep and rest (paired $t$ test, $p=0.0849, n=12$ session pairs on 6 rats, $\mathrm{df}=11$, $t=-1.89$; Fig. $6 A-C$; mean rates: paired $t$ test, $p=0.2915, n=6$, $\mathrm{df}=5, t=-1.17$; mean ratios: $t$ test, $p=0.4035, n=6, \mathrm{df}=5$, $t=0.91$ ), confirming a role for NMDA receptor-dependent processes during learning on the maze. To rule out a possible confounding action of MK-801 during post-learning sleep and rest, in a control experiment the blocker was injected after training (Fig. 6D). As in drug-free animals, interfering with ripples again induced an increase in SPW-R activity (paired $t$ test, $p=0.0059, n=11$ session pairs on 5 rats, $\mathrm{df}=10, t=-3.48$; Fig. $6 D-F$; mean rates: paired $t$ test, $p=0.0418, n=4$, df $=3$, $t=-3.42$, one outlier; mean ratios: $t$ test, $p=0.0319, n=4$, df $=3$, $t=3.80$, one outlier). This confirmed that the suppressive effect 
A

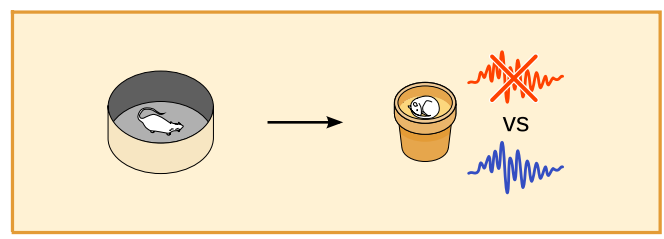

B

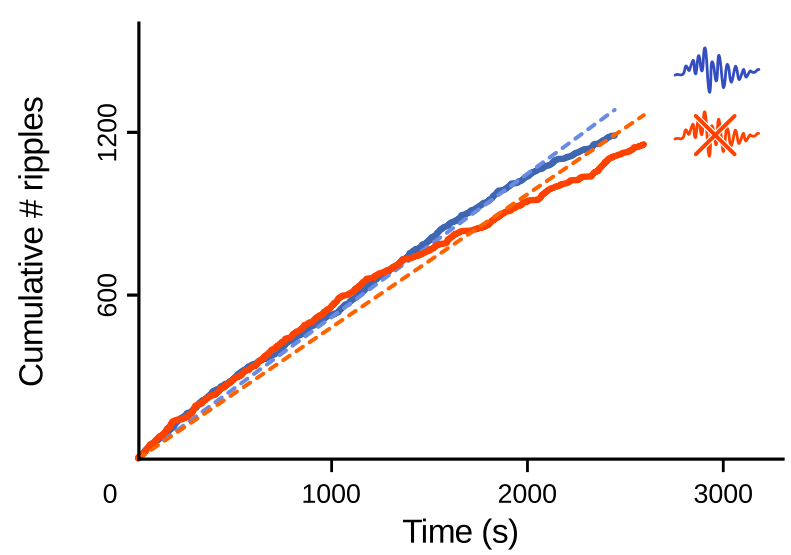

C

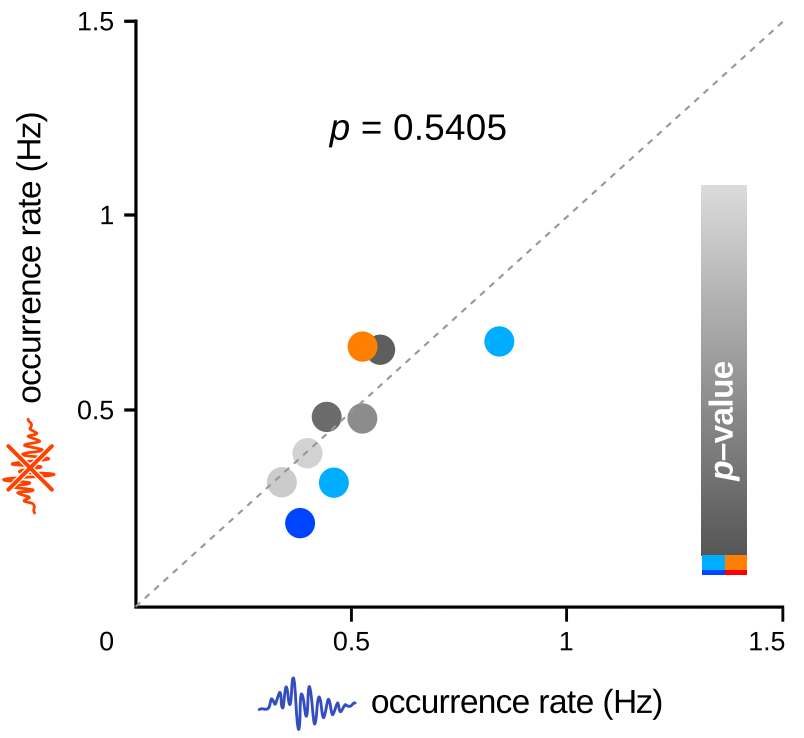

Figure 5. $\quad A$, In the control condition, rats foraged for food in a familiar arena before sleep and rest sessions. $\boldsymbol{B}$, Ripple counts after an example foraging session pair. $\boldsymbol{C}$, Following random foraging in the familiar arena, ripple occurrence rates were not significantly different (paired $t$ test, $p=0.5405, n=11$ ) whether ripples were suppressed ( $y$-axis) or left intact ( $x$-axis).

of MK-801 was selectively due to its action during learning on the radial maze.

\section{Discussion}

Although sleep ripples and associated replay of awake neuronal activity patterns have been associated with spatial memory consolidation (Buzsáki, 1989; Girardeau et al., 2009; Ego-Stengel et al., 2010), how this is dynamically regulated by learning and consolidation requirements remains poorly understood. SPW-Rs are characteristic patterns of hippocampal activity during SWS and quiet rest, whether these states follow complex learning behaviors or mere repetitive locomotor activity. Similarly, replay of awake neural patterns can be observed even in overtrained animals performing simple routine behaviors that are not expected to require consolidation. However, the rate of SPW-R and associated replay were shown to be increased by experimental induction of synaptic plasticity (Buzsáki, 1984; Behrens et al., 2005), as well as training on a learning task (Kudrimoti et al., 1999; Axmacher et al., 2008; Ramadan et al., 2009). The incidence of SPW-Rs and replay events could therefore constitute an important factor of effective consolidation. This raises the question of whether ripples following learning are subject to different regulatory mechanisms that would be initiated during awake behavior, possibly by specific plasticity and tagging processes occurring during learning.

Here, we selectively interfered with memory consolidation processes by systematically suppressing ripple activity, and showed that this triggered immediate increased SPW-R activity following training on a spatial reference memory task, but not random foraging in a familiar environment. Critically, the experiments were carefully designed to minimize potential spurious differences due to intraindividual and interindividual variability in ripple occurrence and detection rates. Thus, each animal was used as its own control across stimulation protocols and behavioral conditions (Fig. 1A-C). Furthermore, stimulation protocols and behavioral conditions were alternated in a pseudorandom order across days and animals (Fig. 1D). This experimental design was instrumental in ensuring reliable and comparable measurements across experimental conditions. Although this prevented the quantification of daily changes in task performance on the radial maze, it was previously established that ripple suppression, but not delayed stimulation, impairs memory consolidation on this and similar spatial reference memory tasks (Girardeau et al., 2009; Ego-Stengel and Wilson, 2010). We then interfered with initial plasticity mechanisms set up during learning, and showed that NMDA receptor-dependent processes were instrumental in setting up the observed increased SPW-R drive. NMDA receptors are crucial for hippocampal LTP at CA synapses (Collingridge et al., 1983), and instrumental for spatial memory (Morris et al., 1986; Tsien et al., 1996; Shimizu et al., 2000; Nakazawa et al., 2004; Dupret et al., 2010), although it remains unclear whether they are necessary during acquisition, consolidation, or both. Several studies suggest that NMDA receptor activation during acquisition or initial exploration are important for subsequent consolidation. For instance, NMDA receptors are instrumental for the long-term stability of newly formed place fields, but not for already established place fields (Kentros et al., 1998). It was also shown that NMDA receptor blockade does not impair acquisition during massed trials, but prevents subsequent consolidation by impairing goal-related place cell reactivations of the preceding trials (but not of older, drug-free trials; Dupret et al., 2010; Santini et al., 2001). Hence, while NMDA receptor blockade during sleep does not prevent hippocampal replay, NMDA-receptor blockade during exploration impairs subsequent replay during sleep (Dupret et al., 2010). It was also shown that systemic injections of the NMDA receptor blocker CPP [3-(2-carboxypiperazin-4-yl)propyl-1-phosphonic acid] before, but not after, training on an object displacement task impaired performance on subsequent testing (Larkin et al., 2008). Consistent with a role of NMDA receptors in setting up network and cellular mechanisms for subsequent consolidation, we showed that blocking hippocampal and cortical NMDA receptors by systemic injection of MK-801 before training suppressed the subsequent increase in SPW-R drive. Interestingly, this suppressive effect was specifically triggered by NMDA receptor activation during training but not during sleep, as posttraining injections of $\mathrm{MK}-801$ did not affect the up-regulation. This is also consistent with an in vitro study showing that experimental LTP induction triggers sustained ripple activity in slices 


\section{A}

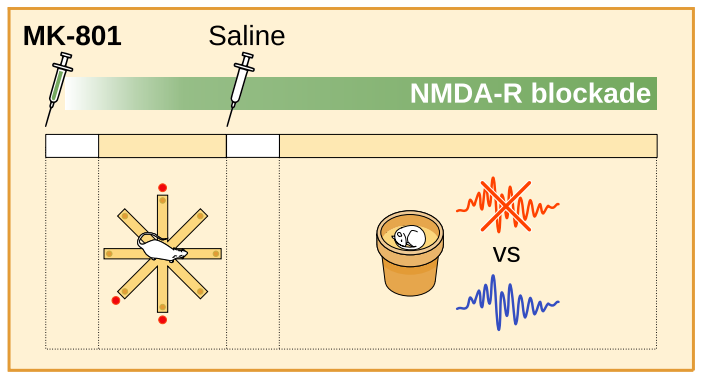

B

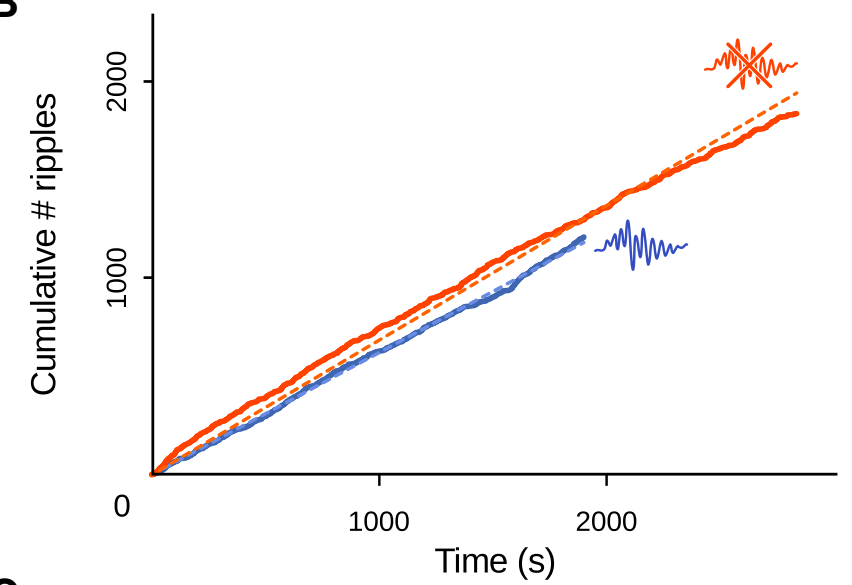

C

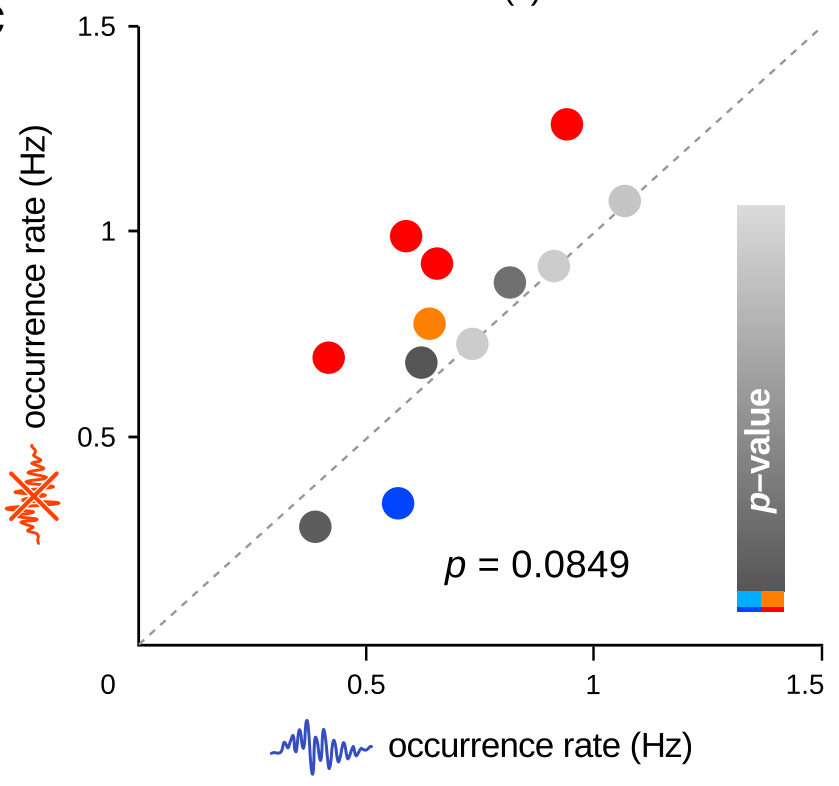

D

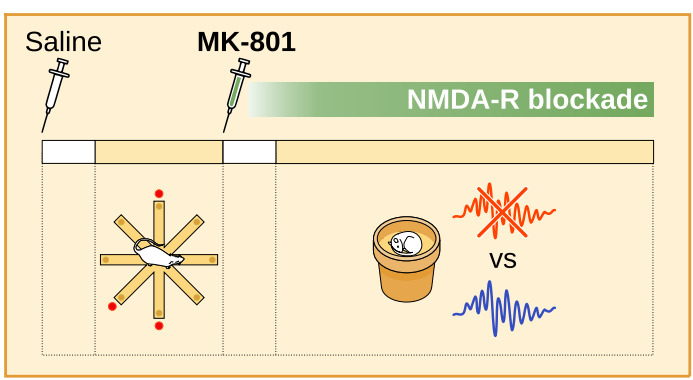

$\mathbf{E}$

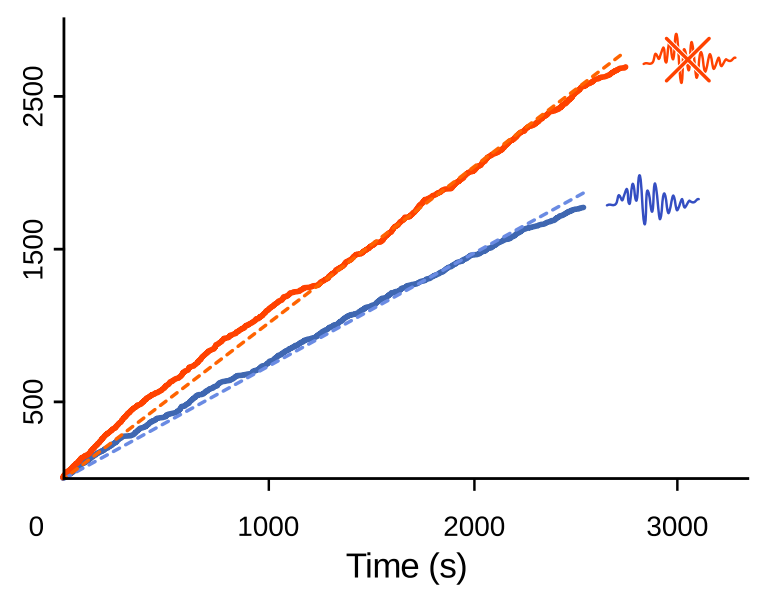

F

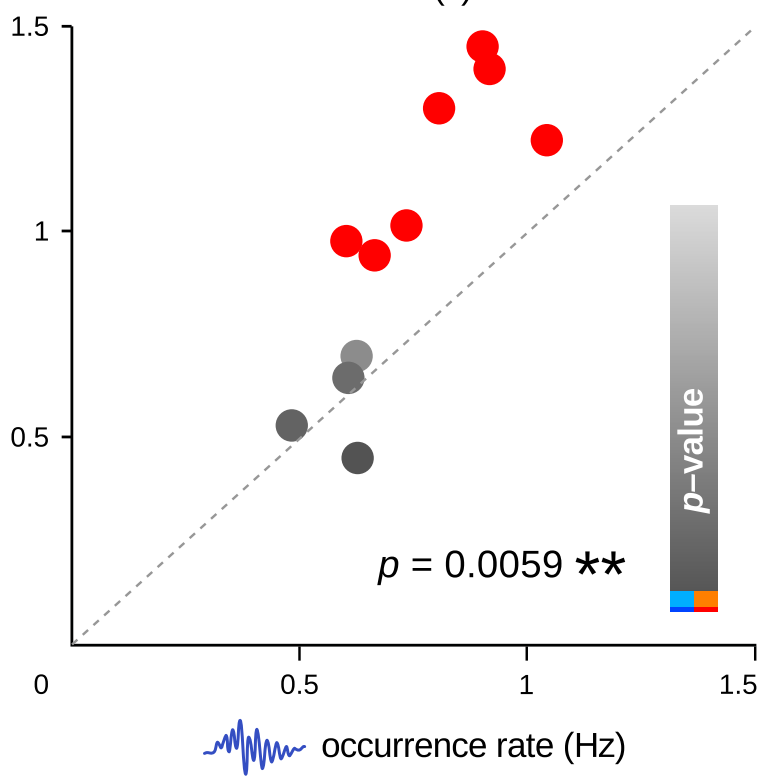

Figure 6. NMDA receptor blockade during training abolishes SPW-R upregulation. $\boldsymbol{A}-\boldsymbol{F}$, MK-801 was injected systemically either before $(\boldsymbol{A}-\boldsymbol{C})$ or after $(\boldsymbol{D}-\boldsymbol{F})$ training on the maze (complemented with control saline injections). During subsequent sleep and rest, ripples were either suppressed or left intact. Each injection was followed by a 15 min delay before training or sleep/rest recordings (white rectangles). $\boldsymbol{B}, \boldsymbol{E}$, Ripple counts for an example session pair when NMDA receptors were blocked either during $(\boldsymbol{B})$ or after $(\boldsymbol{E})$ training. $\boldsymbol{C}, \boldsymbol{F}$, SPW-R upregulation is abolished if MK-801 is administered before ( $\boldsymbol{C}$; paired $t$ test, $p=0.0849, n=12$ ) but not after ( $\boldsymbol{F}$; paired $t$ test, $p=0.0059, n=11$ ) training.

lacking spontaneous ripples. Importantly, NMDA receptors were necessary for the induction of ripples, but not for their subsequent expression (Behrens et al., 2005).

We thus propose that the increased drive for SPW-Rs after learning resulted from an NMDA receptor-dependent network plasticity and tagging process initiated during learning and setting the stage for subsequent consolidation during sleep and rest. This would be similar to the synaptic tagging and capture process (Frey and Morris, 1997), but manifested at the network level. A similar process was recently described in orbitofrontal cortex.
Although this structure was not directly involved in the acquisition and early retrieval of a food preference transmission task, NMDA receptor activation was shown to be necessary in the orbitofrontal cortex at the time of encoding for subsequent successful consolidation (Lesburguères et al., 2011). Similarly, here NMDA receptor-dependent plastic changes and tagging of CA3 pyramidal cells initiated during behavior would make a number of "preselected" cells more likely to subsequently entrain each other during sleep and rest. Inputs from tagged neocortical areas may also contribute to the selection process by biasing reactiva- 
tion patterns. Another potential key factor would be the selective activation of neuromodulatory systems both during learning (but not routine exploration) and subsequent sleep. For instance, the dopaminergic and noradrenergic systems are selectively activated during learning, in particular in relation to rewards (Schultz, 2007; Sara and Bouret, 2012), and noradrenergic neurons have been shown to reactivate during SWS following learning tasks (Eschenko and Sara, 2008). These combined influences would result in an increased drive for CA1 ripples (Ramadan et al., 2009) and learning-related replay (Kudrimoti et al., 1999). Candidate mechanisms accounting for the eventual return to baseline would include negative feedback arising from SPW-R activity, possibly involving intrahippocampal as well as hippocampocortical loops (Sirota et al., 2003). Indeed, consolidation is believed to involve a complex hippocampo-cortical dialogue subtended by the coordination of SPW-Rs and cortical rhythms, such as slow oscillations, delta waves, and thalamocortical spindles (Siapas and Wilson, 1998; Sirota et al., 2003; Wierzynski et al., 2009; Sullivan et al., 2011; Hahn et al., 2012; Logothetis et al., 2012). Spindle activity is transiently increased during sleep following learning in both humans and animals, paralleling an increase in hippocampal ripple density (Gais et al., 2002; Eschenko et al., 2006; Mölle et al., 2009). In the medial prefrontal cortex, increases in the reactivation strength of cell assemblies are temporally correlated with hippocampal ripples (Peyrache et al., 2009). We hypothesize that our selective ripple suppression interfered with these delicate dynamic processes, resulting in a compensatory increase in SPW-R initiations. This is consistent with the recent finding that interference with memory consolidation by partial cortical inactivation during learning leads to compensatory hippocampal activation (Lesburguères et al., 2011). Together, our results reveal a selective dynamic regulation of ripple-mediated consolidation of spatial memory following NMDA receptor-dependent tagging and plastic changes occurring during learning.

\section{References}

Axmacher N, Elger CE, Fell J (2008) Ripples in the medial temporal lobe are relevant for human memory consolidation. Brain 131:1806-1817. CrossRef Medline

Behrens CJ, van den Boom LP, de Hoz L, Friedman A, Heinemann U (2005) Induction of sharp wave-ripple complexes in vitro and reorganization of hippocampal networks. Nat Neurosci 8:1560-1567. CrossRef Medline

Buzsáki G (1984) Long-term changes of hippocampal sharp-waves following high frequency afferen activation. Brain Res 300:179-182. CrossRef Medline

Buzsáki G (1989) Two-stage model of memory trace formation: a role for "noisy" brain states. Neuroscience 31:551-570. CrossRef Medline

Buzsáki G, Horváth Z, Urioste R, Hetke J, Wise K (1992) High-frequency network oscillation in the hippocampus. Science 256:1025-1027. CrossRef Medline

Carr MF, Jadhav SP, Frank LM (2011) Hippocampal replay in the awake state: a potential substrate for memory consolidation and retrieval. Nat Neurosci 14:147-153. CrossRef Medline

Collingridge GL, Kehl SJ, McLennan H (1983) Excitatory amino acids in synaptic transmission in the schaffer collateral-commissural pathway of the rat hippocampus. J Physiol 334:33-46. Medline

Csicsvari J, Hirase H, Mamiya A, Buzsáki G (2000) Ensemble patterns of hippocampal CA3-CA1 neurons during sharp wave-associated population events. Neuron 28:585-594. CrossRef Medline

Davidson TJ, Kloosterman F, Wilson MA (2009) Hippocampal replay of extended experience. Neuron 63:497-507. CrossRef Medline

Diba K, Buzsáki G (2007) Forward and reverse hippocampal place-cell sequences during ripples. Nat Neurosci 10:1241-1242. CrossRef Medline

Diba K, Buzsáki G (2008) Hippocampal network dynamics constrain the time lag between pyramidal cells across modified environments. J Neurosci 28:13448-13456. CrossRef Medline
Dragoi G, Buzsáki G (2006) Temporal encoding of place sequences by hippocampal cell assemblies. Neuron 50:145-157. CrossRef Medline

Dupret D, O’Neill J, Pleydell-Bouverie B, Csicsvari J (2010) The reorganization and reactivation of hippocampal maps predict spatial memory performance. Nat Neurosci 13:995-1002. CrossRef Medline

Ego-Stengel V, Wilson MA (2010) Disruption of ripple-associated hippocampal activity during rest impairs spatial learning in the rat. Hippocampus 20:1-10. CrossRef Medline

Eschenko O, Sara SJ (2008) Learning-dependent, transient increase of activity in noradrenergic neurons of locus coeruleus during slow wave sleep in the rat: brain stem-cortex interplay for memory consolidation? Cereb Cortex 18:2596-2603. CrossRef Medline

Eschenko O, Mölle M, Born J, Sara SJ (2006) Elevated sleep spindle density after learning or after retrieval in rats. J Neurosci 26:12914-12920. CrossRef Medline

Eschenko O, Ramadan W, Mölle M, Born J, Sara SJ (2008) Sustained increase in hippocampal sharp-wave ripple activity during slow-wave sleep after learning. Learn Mem 15:222-228. CrossRef Medline

Foster DJ, Wilson MA (2006) Reverse replay of behavioral sequences in hippocampal place cells during the awake state. Nature 440:680-683. CrossRef Medline

Frey U, Morris RG (1997) Synaptic tagging and long-term potentiation. Nature 385:533-536. CrossRef Medline

Gais S, Mölle M, Helms K, Born J (2002) Learning-dependent increases in sleep spindle density. J Neurosci 22:6830-6834. Medline

Girardeau G, Zugaro M (2011) Hippocampal ripples and memory consolidation. Curr Opin Neurobiol 21:452-459. CrossRef Medline

Girardeau G, Benchenane K, Wiener SI, Buzsáki G, Zugaro MB (2009) Selective suppression of hippocampal ripples impairs spatial memory. Nat Neurosci 12:1222-1223. CrossRef Medline

Hahn TT, McFarland JM, Berberich S, Sakmann B, Mehta MR (2012) Spontaneous persistent activity in entorhinal cortex modulates corticohippocampal interaction in vivo. Nat Neurosci 15:1531-1538. CrossRef Medline

Hazan L, Zugaro M, Buzsáki G (2006) Klusters, NeuroScope, NDManager: a free software suite for neurophysiological data processing and visualization. J Neurosci Meth 155:207-216. CrossRef

Jadhav SP, Kemere C, German PW, Frank LM (2012) Awake hippocampal sharp-wave ripples support spatial memory. Science 336:1454-1458. CrossRef Medline

Kentros C, Hargreaves E, Hawkins RD, Kandel ER, Shapiro M, Muller RV (1998) Abolition of long term stability of new hippocampal place cell maps by NMDA receptor blockade. Science 280:2121-2126. CrossRef Medline

Kudrimoti HS, Barnes CA, McNaughton BL (1999) Reactivation of hippocampal cell assemblies: effects of behavioral state, experience, and EEG dynamics. J Neurosci 19:4090-4101. Medline

Larkin AE, Fahey B, Gobbo O, Callaghan CK, Cahill E, O'Mara SM, Kelly AM (2008) Blockade of NMDA receptors pre-training, but not post-training impairs object displacement learning in the rat. Brain Res 1199:126-132. CrossRef Medline

Lee AK, Wilson MA (2002) Memory of sequential experience in the hippocampus during slow wave sleep. Neuron 36:1183-1194. CrossRef Medline

Lesburguères E, Gobbo OL, Alaux-Cantin S, Hambucken A, Trifilieff P, Bontempi B (2011) Early tagging of cortical networks is required for the formation of enduring associative memory. Science 331:924-928. CrossRef Medline

Logothetis NK, Eschenko O, Murayama Y, Augath M, Steudel T, Evrard HC, Besserve M, Oeltermann A (2012) Hippocampal-cortical interaction during periods of subcortical silence. Nature 491:547-553. CrossRef Medline

Marr D (1971) Simple memory: a theory for archicortex. Phil Trans R Soc B 262:23-81. CrossRef Medline

McLamb RL, Williams LR, Nanry KP, Wilson WA, Tilson HA (1990) MK801 impedes the acquisition of a spatial memory task in rats. Pharmacol Biochem Behav 37:41-45. CrossRef Medline

Memmesheimer RM (2010) Quantitative prediction of intermittent highfrequency oscillations in neural networks with supralinear dendritic interactions. Proc Natl Acad Sci U S A 107:11092-11097. CrossRef Medline Mölle M, Eschenko O, Gais S, Sara SJ, Born J (2009) The influence of learn- 
ing on sleep slow oscillations and associated spindles and ripples in humans and rats. Eur J Neurosci 29:1071-1081. CrossRef Medline

Morris RG, Anderson E, Lynch GS, Baudry M (1986) Selective impairment of learning and blockade of long-term potentiation by an N-methyl-Daspartate receptor antagonist, AP5. Nature 319:774-776. CrossRef Medline

Nádasdy Z, Hirase H, Czurkó A, Csicsvari J, Buzsáki G (1999) Replay and time compression of recurring spike sequences in the hippocampus. J Neurosci 19:9497-9507. Medline

Nakazawa K, McHugh TJ, Wilson MA, Tonegawa S (2004) NMDA receptors, place cells and hippocampal spatial memory. Nat Rev Neurosci 5:361-372. CrossRef Medline

O'Keefe J, Dostrovsky J (1971) The hippocampus as a spatial map. Preliminary evidence from unit activity in the freely-moving rat. Brain Res 34: 171-175. CrossRef Medline

Peyrache A, Khamassi M, Benchenane K, Wiener SI, Battaglia FP (2009) Replay of rule-learning related neural patterns in the prefrontal cortex during sleep. Nat Neurosci 12:919-926. CrossRef Medline

Ramadan W, Eschenko O, Sara SJ (2009) Hippocampal sharp wave/ripples during sleep for consolidation of associative memory. PLoS One 4:e6697. CrossRef Medline

Santini E, Muller RU, Quirk GJ (2001) Consolidation of extinction learning involving transfer from NMDA-independent to NMDA-dependent memory. J Neurosci 21:9009-9017. Medline

Sara SJ, Bouret S (2012) Orienting and reorienting: the locus coeruleus mediates cognition through arousal. Neuron 76:130-141. CrossRef Medline

Schomburg EW, Anastassiou CA, Buzsáki G, Koch C (2012) The spiking component of oscillatory extracellular potentials in the rat hippocampus. J Neurosci 32:11798-11811. CrossRef Medline

Schultz W (2007) Behavioral dopamine signals. Trends Neurosci 30:203210. CrossRef Medline
Shimizu E, Tang YP, Rampon C, Tsien JZ (2000) NMDA receptordependent synaptic reinforcement as a crucial process for memory consolidation. Science 290:1170-1174. CrossRef Medline

Siapas AG, Wilson MA (1998) Coordinated interactions between hippocampal ripples and cortical spindles during slow-wave sleep. Neuron 21:1123-1128. CrossRef Medline

Sirota A, Csicsvari J, Buhl D, Buzsáki G (2003) Communication between neocortex and hippocampus during sleep in rodents. Proc Natl Acad Sci U S A 100:2065-2069. CrossRef Medline

Skaggs WE, McNaughton BL (1996) Replay of neuronal firing sequences in rat hippocampus during sleep following spatial experience. Science 271: 1870-1873. CrossRef Medline

Skaggs WE, McNaughton BL, Wilson MA, Barnes CA (1996) Theta phase precession in hippocampal neuronal populations and the compression of temporal sequences. Hippocampus 6:149-172. CrossRef Medline

Sullivan D, Csicsvari J, Mizuseki K, Montgomery S, Diba K, Buzsáki G (2011) Relationships between hippocampal sharp waves, ripples, and fast gamma oscillation: influence of dentate and entorhinal cortical activity. J Neurosci 31:8605-8616. CrossRef Medline

Tsien JZ, Huerta PT, Tonegawa S (1996) The essential role of hippocampal CA1 NMDA receptor-dependent synaptic plasticity in spatial memory. Cell 87:1327-1338. CrossRef Medline

Wierzynski CM, Lubenov EV, Gu M, Siapas AG (2009) State-dependent spike-timing relationships between hippocampal and prefrontal circuits during sleep. Neuron 61:587-596. CrossRef Medline

Wilson MA, McNaughton BL (1993) Dynamics of the hippocampal ensemble code for space. Science 261:1055-1058. CrossRef Medline

Ylinen A, Bragin A, Nádasdy Z, Jandó G, Szabó I, Sik A, Buzsáki G (1995) Sharp wave-associated high-frequency oscillation $(200 \mathrm{~Hz})$ in the intact hippocampus: network and intracellular mechanisms. J Neurosci 15:30-46. Medline 